La Revue

des Droits

de l'Homme

\section{La Revue des droits de l'homme}

Revue du Centre de recherches et d'études sur les droits fondamentaux

Actualités Droits-Libertés | 2018

\title{
Traité franco-britannique de Sandhurst : tout changer pour ne rien changer
}

Droit des étrangers (Traité de Sandhurst)

\section{Antoine Guérin}

\section{(2) OpenEdition}

\section{Journals}

Édition électronique

URL : http://journals.openedition.org/revdh/3772

DOI : $10.4000 /$ revdh.3772

ISSN : 2264-119X

Éditeur

Centre de recherches et d'études sur les droits fondamentaux

Référence électronique

Antoine Guérin, «Traité franco-britannique de Sandhurst : tout changer pour ne rien changer », La Revue des droits de l'homme [En ligne], Actualités Droits-Libertés, mis en ligne le 30 juin 2018, consulté le 03 mai 2019. URL : http://journals.openedition.org/revdh/3772 ; DOI : 10.4000/revdh.3772

Ce document a été généré automatiquement le 3 mai 2019.

Tous droits réservés 


\title{
Traité franco-britannique de Sandhurst : tout changer pour ne rien changer
}

\author{
Droit des étrangers (Traité de Sandhurst)
}

\section{Antoine Guérin}

\author{
" [...] la frontière vers l'Angleterre ne peut être franchie [...]. » \\ Éric Besson, ministre de l'Intérieur, 23 avril 2009 \\ « La frontière à Calais est fermée et à vocation à le demeurer. » \\ Bernard Cazeneuve, ministre de l'Intérieur, 29 juin 2016 \\ "Si on veut aller en Grande-Bretagne, ce n'est pas à Calais qu'il faut venir. » \\ Gérard Collomb, ministre de l'Intérieur, 2 février 2018
}

1 Le 18 janvier dernier, le Premier ministre britannique Theresa May et le président de la République français Emmanuel Macron se sont retrouvés à Sandhurst, dans le comté de Berkshire, au sud-ouest de Londres, pour un événement rituel auquel aucun chef de gouvernement britannique ou chef d'État français n'échappe : le sommet francobritannique. Il est souvent, si ce n'est toujours, l'occasion pour l'un et pour l'autre de se féliciter de la longévité et de la qualité de la relation entre le Royaume-Uni et la France, de commémorer l'alliance de ces derniers lors des deux guerres mondiales et, bien sûr, d'apposer leur signature solennelle, ou celle de leurs ministres, sur quelques traités de partenariat.

2 Mis à part le fait d'avoir été tous les quatre ministres de l'Intérieur, Jean-Pierre Chevènement, Nicolas Sarkozy, Éric Besson et Bernard Cazeneuve ont un autre point commun : tous ont signé, au nom du gouvernement français, un texte capital relatif à la gestion de la frontière franco-britannique. L'actuel ministre de l'Intérieur n'a pas échappé à la règle : fraîchement nommé " numéro 2 » du gouvernement en mai 2017, Gérard Collomb a signé, moins d'un an plus tard lors de ce 35e sommet franco-britannique, aux côtés de son homologue Amber Rudd, un texte intitulé Traité entre le gouvernement du Royaume-Uni de Grande-Bretagne et d'Irlande du Nord et le gouvernement de la République française relatif au renforcement de la coopération pour la gestion coordonnée de leur frontière 
commune, communément appelé "traité de Sandhurst », entré en vigueur le 1er février dernier.

3 Ce texte s'ajoute à la longue liste des traités, protocoles et arrangements conclus entre le Royaume-Uni et la France depuis 1986. Au nombre de dix-neuf, tous poursuivent un but principal : restreindre, si ce n'est empêcher, l'accès des exilés au territoire britannique. Toujours plus rigoureux et coercitifs, indistinctement de l'étiquette politique du gouvernement qui les signe, ces textes aux contours légaux relativement flous sont à l'origine d'un régime juridique ad hoc qui concurrence voire neutralise les législations nationales, européennes et internationales en matière de droit des migrations et de droits de l'homme.

La victoire d'Emmanuel Macron, positionné politiquement comme «ni de droite, ni de gauche $»^{1}$, à l'élection présidentielle française, fut l'occasion pendant un certain temps de laisser croire que la situation migratoire à Calais pourrait connaître un autre dessein. Alors ministre de l'Économie, Emmanuel Macron avait en effet affirmé que si le peuple britannique se prononçait majoritairement en faveur du «Leave » lors du référendum du 23 juin 2016, « les migrants ne ser[aien]t déjà plus à Calais »². À plus forte raison, selon ses dires, s'il était élu président de la République, le traité du Touquet, pierre angulaire tant décriée de cette coopération bilatérale, serait renégocié. Cette proposition, déjà faite quelques mois auparavant par Nicolas Sarkozy - celui-là même qui avait signé le traité du Touquet -, avait provoqué l'ire du ministre de l'Intérieur de l'époque, Bernard Cazeneuve, qui avait accusé son collègue du gouvernement de vouloir faire le «buzz ». Celui qui était alors en charge du dossier lui avait sèchement répondu que la question «ne se pos[ait] pas " ${ }^{3}$, alors que François Hollande lui-même avait fait une proposition en ce sens, avant de se raviser.

Deux mois avant le premier tour de l'élection présidentielle, à la suite de sa rencontre avec le Premier ministre Theresa May, Emmanuel Macron apporta une nuance considérable à ses déclarations premières, affirmant vouloir réviser les accords du Touquet «sans tout casser» et suggérant de «rééquilibrer la relation pour que [...] la contribution britannique soit revue à la hausse $»^{4}$. Si le doute concernant ses intentions n'était plus permis à l'aune de ce revirement soudain, la presse a laissé l'opinion en suspens en rapportant les propos de Theresa May qui, commentant la victoire d'Emmanuel Macron, garantissait que «le gouvernement britannique [allait] se mettre à la table des négociations avec Monsieur Macron et les autres pour discuter du fonctionnement du système existant au profit du Royaume-Uni et de la France $»^{5}$. Malheureusement moins tapageur que l'abandon du projet de l'aéroport de Notre-Damedes-Landes, le traité de Sandhurst est pourtant venu mettre un terme à cette saynète médiatique au scénario écrit à l'avance. Fidèle à la trajectoire dessinée par les deux États depuis les années 1990, le traité de Sandhurst confirme que ni le temps, ni les circonstances n'ont d'emprise sur la politique migratoire menée dans ce cadre, témoignant ainsi de son dogmatisme.

6 Le traité de Sandhurst valide et sacralise tous les codes et les fondements établis quinze ans plus tôt par le traité du Touquet (I). Nonobstant, il apporte un éclairage non négligeable, bien que très équivoque, quant à l'évolution de la coopération francobritannique ainsi qu'aux relations entre le Royaume-Uni et l'Union européenne à la suite du Brexit (II). 


\section{$1 \%$ - Une coopération immuable malgré d'apparents changements}

7 "Changer en profondeur les choses ", « une avancée majeure ", «mettre un terme à la situation $»^{6} .$. les termes employés lors de la conférence de presse de Theresa May et d'Emmanuel Macron à propos du traité de Sandhurst sont prometteurs, en dépit d'une résonance familière. En effet, ils sont identiques à ceux employés lors de la signature des accords précédents. Si la forme est toujours la même, le contenu n'en est pas moins identique ; les services de l'Élysée avaient d'ailleurs signifié à la presse quelques heures avant sa signature que ce nouveau texte visait à "renforcer ", " compléter » le traité du Touquet. Le traité de Sandhurst est le nouveau nom d'un dispositif dissuasif qui, lui, n'a pas changé (A) ; s'il a bénéficié d'une relative bienveillance de la part des associations à l'aune de ses mesures concernant les mineurs isolés, elles n'ont certainement pas été accordées de manière gratuite par le Royaume-Uni qui s'est bien gardé d'oublier d'en limiter largement la portée et le champ d'application (B).

\section{A/ - Faire du neuf avec du vieux}

8 De par son intitulé officiel, le traité de Sandhurst se veut plutôt clair. Annoncé lourdement comme «relatif au renforcement de la coopération pour la gestion coordonnée de [la] frontière commune", il clarifie dès son titre la trajectoire que va suivre la politique franco-britannique en la matière : la même que d'habitude. Il est vrai, les mots employés lors des nouveaux accords changent selon les ministres, mais pas leur fond. C'est à partir de $2009^{7}$ que le « renforcement de la frontière commune » va devenir le leitmotiv de la coopération franco-britannique. L'arrangement administratif d'Évian du 6 juillet 2009 avait pour objet de déterminer «les conditions du renforcement de la frontière commune "; l'arrangement administratif de Londres du 2 novembre 2010 se veut «relatif au renforcement de la frontière commune»; en $2014^{8}$, les mots « renforcer » et « renforcement » sont rebattus, la déclaration conjointe du 20 septembre affirmant l'objectif de "renforcer la sécurité » de la frontière et de «renforcer la coopération opérationnelle "; la déclaration commune du 20 août 2015 affiche son but "renforcer la sécurité » de la frontière et de "renforcer encore davantage [la] collaboration opérationnelle "; la déclaration conjointe sur les migrations du 3 mars 2016 a pour souci de "renforcer la sécurité aux frontières" et le "renforcement de la coopération"; en $2017^{9}$, les mots ont peu changé, mais le fond pas du tout : le communiqué de presse conjoint du 16 novembre 2017 parle de «renforcement des aménagements de sécurité », de " poursuivre une coopération étroite et constructive » et de "gestion conjointe de la frontière commune", formule que l'on retrouve dans l'intitulé du traité de Sandhurst.

Depuis son titre, le traité de Sandhurst reprend ce qu'on pourrait appeler les " caractéristiques » d'un accord franco-britannique en matière de politique migratoire. Pour son entrée en matière, chose nouvelle, le Traité expose ses visas : les deuxième et troisième paragraphes commencent tous deux par la mention "Having regard", qui indique sur quels textes juridiquement supérieurs ou principes se fonde la décision. En l'occurrence, le deuxième paragraphe désigne les deux premiers fondements du Traité : le traité de Cantorbéry du 12 février 1986, qui autorise le commencement des travaux du 
tunnel sous la Manche, et le protocole de Sangatte du 25 novembre 1991. Premier problème, l'incompatibilité du protocole de Sangatte avec le droit de l'Union européenne - notamment relatif à l'espace Schengen - est indiscutable ${ }^{10}$. Compilé à son protocole additionnel, ce protocole institue des contrôles drastiques dans les gares ferroviaires britanniques et françaises pour éviter toute intrusion indésirable lors du départ des trains à destination du Royaume-Uni ; le traité du Touquet, signé le 4 février 2003, bénéficie quant à lui d'un paragraphe qui lui est réservé. Second problème, ce traité a appliqué ces mêmes contrôles aux ports maritimes britanniques et français et institué une politique de migration et d'asile parallèle à celle de l'Union européenne, la seconde étant pourtant censée primer sur la première. Depuis l'entrée en vigueur du traité du Touquet, les systèmes Schengen et Dublin se retrouvent neutralisés et la frontière britannique s'est officiellement déplacée sur le territoire français. La quatrième et dernière référence textuelle est la déclaration commune du 20 août 2015, dont le but principal affiché était d'augmenter considérablement le nombre de retours d'exilés dans leur pays d'origine.

10 Le traité de Sandhurst, comme nul autre accord avant lui, avalise et légitime les accords précités comme des sources inébranlables, des "textes fondateurs", un «bloc de constitutionnalité " de la coopération franco-britannique. Largement ancré dans ce cadre, il n'a donc pas pour objet de rectifier la politique de contournement des lois européennes et internationales des deux pays en matière migratoire. S'appuyant sur des actes extralégaux, c'est-à-dire qui sont contraires au droit de l'Union et au droit international mais qui n'ont jamais été sanctionnés comme tels, le Traité se retrouve donc dépourvu de base légale. Il s'inscrit pourtant dans ce régime juridique parallèle institué par les accords Sangatte-Touquet que les deux États admettent n'avoir absolument pas l'intention de remettre en cause. Le Traité précise en effet : « Les deux Parties réaffirment leur attachement au fonctionnement efficace et durable de leur frontière commune sur la base du cadre juridique établi pour sa sécurité par les accords susmentionnés [...] ».

11 Le préambule du Traité s'ensuit par un constat : «Alors qu'un an après le démantèlement du camp de la Lande à Calais, la persistance d'une forte pression migratoire a conduit les Parties à réaffirmer leur volonté commune d'une coopération étroite dans la gestion de leur frontière commune [...].» Ce constat, inédit pour un traité du genre, retentit davantage comme un aveu d'échec : il apprend au lecteur que la seule motivation factuelle à la signature de ce nouveau traité (il n'y en a effectivement pas d'autres revendiquées dans ce préambule) tient au fait qu'un an après le démantèlement de la " "nouvelle jungle" de Calais », la situation n'a pas évolué ; l'histoire s'est de nouveau répétée. Pourtant, il faut rappeler que des mesures étaient censées avoir été prises et que des garanties que la situation ne se reproduirait pas avaient été données par Theresa May elle-même, à l'époque secrétaire d'État à l'Intérieur, et Bernard Cazeneuve, à l'époque ministre de l'Intérieur, ancien collègue de gouvernement d'Emmanuel Macron sous la présidence de François Hollande. Cet aveu d'échec révèle l'existence de ce cercle vicieux qu'est la politique de coopération franco-britannique : le phénomène migratoire a entraîné la répression ; inefficace, le phénomène migratoire s'est reproduit ; la répression s'est amplifiée ; inefficace, le phénomène s'est reproduit, et ainsi de suite.

12 L'article 1 du Traité présente trois engagements conjoints de la part des gouvernements britannique et français. À leur lecture, il s'avère que ces trois engagements n'en forment en réalité qu'un seul, que l'on retrouve toujours au fil des accords et des traités bilatéraux contemporains. Le premier, à la formule non dénuée de cynisme, consiste à «accroître 
davantage la coopération en matière de gestion de [la] frontière commune et réduire le nombre de personnes qui tentent de la franchir illégalement et qui courent des risques pour leur sécurité et leur vie ». S'il est inutile d'expliquer en quoi franchir la frontière n'est pas dangereux en soi mais que le danger est fabriqué et issu d'une réelle volonté politique, le premier engagement est donc le suivant : réduire le nombre de tentatives de franchissement de la frontière.

Par la mise à disposition d'hébergements situés en dehors de Calais et de Dunkerque, l'amélioration du transfert des demandeurs d'asile et l'augmentation d'un nombre "sensiblement accru» de retours d'exilés dans leur pays d'origine, le deuxième engagement consiste à « réduire la pression migratoire à la frontière commune et du côté français de la Manche et de la mer du Nord ». Clair dans sa formulation et reconnaissant par là même que le système Sangatte-Touquet, ainsi que le présent Traité, n'induisent absolument pas des engagements réciproques de la part des deux États, le deuxième engagement consiste in fine à la même chose que le premier : réduire le nombre de tentatives de franchissement de la frontière.

Le troisième et dernier engagement, énoncé en des termes très généraux, confirme le but recherché : «Mettre en œuvre, conformément à la législation applicable, un programme de mesures spécifiques convenues d'un commun accord pour lutter contre les réseaux criminels organisés, la fraude et les mouvements illégaux de marchandises et de personnes, et pour décourager l'immigration clandestine, par une action conjointe dans les pays d'origine et de transit. » L'objectif est encore le même : réduire le nombre de tentatives de franchissement de la frontière. Cette "action conjointe dans les pays d'origine et de transit ", euphémisme pour ne pas dire "politique de retours ", prend une place particulièrement importante dans le Traité. S'il ne lui est pas assigné d'objectifs chiffrés, elle se retrouve dans différentes parties du texte, parfois en filigrane comme dans l'article $1 \mathrm{ou}$, plus étonnant, dans l'article 4, qui est certainement le plus incohérent de tous.

Intitulé « Protection des demandeurs d'asile », l'article 4 commence en ces termes : «Les deux Parties s'engagent à faciliter, sur leur territoire respectif, l'accès à la procédure d'asile pour ceux qui sollicitent une protection internationale.» Si cet objectif semble louable, il doit être lu à la lumière du système Sangatte-Touquet. Premièrement, comme l'a rappelé Gérard Collomb, la frontière franco-britannique est fermée, et les 50 millions d'euros versés par le Royaume-Uni à la France - dont près du tiers sera consacré à la vidéosurveillance, aux barrières de sécurité et aux équipes cynophiles ${ }^{11}$ - veilleront à ce qu'elle le reste ; si les exilés n'ont pas la possibilité d'accéder au territoire du RoyaumeUni, ils n'ont évidemment pas la possibilité d'y déposer une demande d'asile. De plus, le système Sangatte-Touquet, en vertu duquel les demandes d'asile déposées auprès des autorités britanniques dans les gares ferroviaires et les ports maritimes français doivent être étudiées par la France, est conforté par le traité de Sandhurst. Les exilés n'ont en conséquence ni la possibilité de déposer une demande d'asile sur le territoire britannique, ni celle de la déposer auprès des autorités britanniques dans les gares et ports français. L'accès à la procédure d'asile n'est absolument pas «facilitée " par le Royaume-Uni à travers le traité de Sandhurst. Sur ce point, le blocage demeure entier.

16 Si le traité de Sandhurst a donc une fonction de trompe-l'œil pour mieux confirmer la perdurance du système Sangatte-Touquet, les mesures concernant les mineurs ont pratiquement éludé toutes les autres. Présentées comme une victoire d'Emmanuel Macron dans les négociations, elles suscitent cependant une circonspection certaine, à 
raison du peu de précisions qu'elles contiennent et du marchandage dont elles semblent avoir fait l'objet à la lecture du texte du traité.

\section{B/ - Beaucoup de retours contre un peu de mineurs}

17 Si l'on s'en tient à la lettre de l'article 4, le Royaume-Uni soutiendra donc la France pour fournir des hébergements dans les centres d'accueil et d'évaluation des situations (CAES) à ceux qui "sont disposés à s'engager avec le système d'asile en France ». Sont donc exclus ceux qui souhaitent « s'engager avec le système d'asile » au Royaume-Uni ; c'est-àdire l'immense majorité des exilés présents à Calais. Or, d'après les premiers retours d'expérience, les options proposées aux exilés dans ces CAES sont les suivantes : évaluer si une demande d'asile est possible en France, être assigné à résidence ${ }^{12}$ ou placé en rétention administrative ${ }^{13}$ si l'on relève du règlement Dublin III (de par la prise d'empreintes dans un autre pays, en attendant un éventuel transfert), être placé en centre de rétention en attendant une éventuelle expulsion ou accepter l'aide au retour volontaire proposée par l'office français de l'immigration et de l'intégration (OFII). Ce traité augmentera sans doute le nombre de placements en rétention administrative de demandeurs d'asile relevant du règlement Dublin III. La Cour de justice de l'Union européenne a affirmé, dans un arrêt rendu le 15 mars 2017, que les critères objectifs définissant le "risque non négligeable de fuite », qui permet le placement en rétention administrative des "dublinés ", au titre de l'article 28 du règlement Dublin III, doivent être fixés par une loi écrite. Pour la Cour, à défaut de cette loi écrite, le placement en rétention permis par le règlement Dublin III est impossible ${ }^{14}$. Si la législation française ne contient pas de définition du «risque non négligeable de fuite», les préfectures françaises ont pourtant l'habitude de pratiquer ce placement en rétention, en violation de la législation européenne ${ }^{15}$. Le Conseil d'État a validé ce placement en rétention administrative lorsqu'il intervient après la notification de décision de transfert vers un autre État membre ${ }^{16}$. Les parlementaires français sont allés encore plus loin : le Sénat a adopté en première lecture, le 25 janvier 2018, la proposition de loi adoptée par l'Assemblée nationale " permettant une bonne application du régime d'asile européen $»^{17}$ (sic), qui modifie l'article L. 554-1 du Code de l'entrée et du séjour des étrangers et du droit d'asile, autorisant ainsi le placement en rétention administrative d'un demandeur d'asile en attente d'une décision de transfert ${ }^{18}$.

L'article 4 ne promet en aucun cas un quelconque accès au système d'asile français (un grand nombre des exilés ayant transité par un autre pays de l'Union européenne, il sera facile de les y renvoyer ${ }^{19}$ ), et encore moins au système d'asile britannique. Il se termine sur une formule détonnante, affirmant le soutien du Royaume-Uni à la France concernant « les pressions créées par les ressortissants de pays tiers » (sic) «qui n'ont pas de base légale de séjour sur le territoire français et qui sont basés dans les zones autour des ports $\mathrm{du}$ Nord et du Pas-de-Calais ». Reste à savoir en quoi pourrait bien consister ce «soutien ».

19 Ce «soutien» du Royaume-Uni à la France face aux "pressions créées par les ressortissants de pays tiers » trouve sa concrétisation dans l'article 7, intitulé « Mise en œuvre des mesures de retour». Sa formulation expose la nature du soutien prévu à l'article 4. Il s'agit de «renforcer l'efficacité de la politique d'éloignement des personnes sans base légale de séjour sur le territoire français [nos italiques] [...] ». Les ressortissants " sans base légale de séjour sur le territoire français », c'est-à-dire l'immense majorité des 
exilés de Calais qui refusent de déposer une demande d'asile en France par peur d'être renvoyés dans un pays de transit, sont donc, de par le Traité, considérés comme étant en séjour irrégulier et destinés à être renvoyés dans leur pays d'origine. Cette politique de retours est renforcée par le Traité via quatre mesures spécifiques.

La première consiste en « des programmes conjoints pour le retour de ceux qui n'ont pas de base légale de séjour sur le territoire français vers leur pays d'origine ou vers un pays où ils sont légalement admis, y compris par le biais d'opérations conjointes de retour par voie aérienne ». Déjà en 2009, l'arrangement administratif d'Évian instaurait cette politique de retours, en donnant pour objectif à la coopération franco-britannique d' " augmenter de manière significative le nombre de retours forcés d'étrangers en situation irrégulière et leur réintégration dans leurs pays d'origine ou de transit $»^{20}$. L'accord du 20 août 2015 prévoyait également d' "augmenter le nombre d'éloignements de migrants illégaux dans leurs pays", de "garantir le maximum de résultats" et que le gouvernement britannique "apportera[it] une contribution financière et un appui en matière de vols retour ${ }^{21}$. Cet objectif n'a pas changé. Le traité de Sandhurst, en son article 1, précise explicitement que ces retours doivent concerner un nombre « sensiblement accru » de ressortissants de pays tiers. Reste à savoir si ces retours seront forcés ou volontaires. Dans le premier cas, s'ils sont collectifs, ils sont interdits par la Convention européenne des droits de l'homme à son article 4. La Cour européenne des droits de l'homme, par plusieurs arrêts, a déjà condamné la pratique des retours forcés lorsqu'ils consistent en des refoulements indiscriminés et automatiques, sans examen de situation personnelle ${ }^{22}$. Saisie les 11 et 14 novembre 2008 de onze requêtes déposées par des ressortissants afghans qui attendaient d'être renvoyés dans leur pays par un vol franco-britannique, la Cour européenne des droits de l'homme avait déjà adressé une injonction à la France de renoncer à ces expulsions qui auraient pu exposer ces individus à des risques réels de torture ou de mauvais traitements ${ }^{23}$.

21 La deuxième mesure prévue par l'article 7 concerne « la mise à disposition d'un service d'interprétation disponible en permanence en mettant en commun [les] ressources d'interprétation dans des langues rares telles que le kurde, le tigrinya, l'oromo et l'amharique ». Cette volonté de se faire comprendre à tout prix concernant le retour pourrait concorder avec le lancement d'un nouveau site Internet par l'OFII, « www.retourvolontaire.fr ", disponible dans pas moins de seize langues, et qui met en avant le parcours de personnes « retournées». On ne peut manquer de relever que, dans le Traité, l'augmentation des effectifs d'interprétariat n'est prévue que concernant la politique des retours.

22 La troisième mesure consiste à «soutenir le développement mutuel des moyens et des accords nécessaires à la réussite des retours ». Cette disposition se trouve concrétisée par un budget de 7 millions d'euros parmi les 50 alloués par le Royaume-Uni à l'occasion de la signature de ce traité ${ }^{24}$.

La quatrième et dernière mesure consiste à renforcer la coopération pour l'identification des personnes sans base légale de séjour sur le territoire français dans le but de les renvoyer vers leur pays d'origine, en prenant contact avec les autorités consulaires en France pour obtenir aux ressortissants concernés des laissez-passer.

Partout dans le Traité, il est question de politique de retours vis-à-vis des ressortissants présents sur le territoire français uniquement, jamais sur le territoire britannique ; preuve une fois encore que ce traité n'a nullement l'intention de rétablir la frontière britannique au Royaume-Uni. De facto, la France va continuer à procéder à des expulsions 
et des retours volontaires pour le compte du Royaume-Uni. Si la politique de retours a toujours occupé une place particulière au sein des accords de coopération francobritanniques, elle semble devenir plus prégnante encore : elle est le revers évident de la médaille de l'accueil supposé de quelques mineurs étrangers par le Royaume-Uni.

Cette réelle concrétisation de la politique de retours apparaît en effet comme un gage donné au Royaume-Uni en échange de sa bonne volonté à l'égard de quelques mineurs étrangers, qui a été saluée çà et là mais qui n'apporte pourtant que peu de solutions nouvelles au problème. Aux termes de l'article 2 du Traité, le Royaume-Uni s'est d'abord engagé à accueillir des mineurs étrangers au titre des dispositions du règlement Dublin visant le regroupement familial. Soit dit en passant, il est étonnant que le Royaume-Uni réaffirme un engagement par lequel il était déjà lié depuis longtemps par le règlement Dublin III. Pour pouvoir espérer gagner le Royaume-Uni grâce au règlement Dublin, comme tout demandeur d'asile, un mineur étranger doit déposer sa demande en préfecture en France. Néanmoins, un mineur étranger doit obligatoirement bénéficier d'un représentant légal pour pouvoir déposer sa demande d'asile, soit par le biais d'une tutelle d'État prononcée par un juge ${ }^{25}$, soit par la désignation d'un administrateur ad hoc par le procureur de la République ${ }^{26}$. L'article 19 de la loi no 2015-925 du 29 juillet 2015 relative à la réforme du droit d'asile prévoit un délai de trois jours pour l'enregistrement de la demande en préfecture, ou un délai de dix jours si un grand nombre de demandeurs présentent leur demande simultanément. Si ces délais semblent courts, le Conseil d'État, dans un arrêt du 21 avril 2017, a affirmé qu'ils n'étaient pas obligatoires ${ }^{27}$. La préfecture envoie ensuite une demande de prise en charge qui doit intervenir dans un délai de trois mois. À ce stade, le Traité ne change rien. Les délais ne sont modifiés qu'au stade de l'étude du dossier par le Home Office britannique, qui a désormais dix jours pour évaluer la situation du mineur, ainsi qu'au stade de l'organisation du transfert : si le Home Office accepte le transfert, la France a désormais quinze jours pour l'organiser. Voilà les seules modifications proposées par le Traité concernant le regroupement familial des mineurs, qui ne donne pas de chiffres concernant le nombre de mineurs qui seront transférés au titre de Dublin ni ne précise comment ils seront identifiés. Fait important, lors du dernier démantèlement de la "jungle », les Britanniques s'étaient engagés à accueillir plusieurs centaines de mineurs sur le fondement de l' "amendement Dubs », mais avaient publié des critères d'éligibilité drastiques pour son application. L'enfant devait d'abord répondre à l'un des critères suivants : avoir douze ans au moins, être orienté par les autorités françaises en raison d'un risque d'exploitation sexuelle, avoir quinze ans ou moins et être de nationalité syrienne ou soudanaise, avoir dix-huit ans ou moins et accompagner une sœur ou un frère qui répond à l'un des critères. Si l'enfant remplissait une de ces conditions, il devait ensuite remplir l'une des trois suivantes : son transfert devait être décidé par le Royaume-Uni en raison de l'intérêt supérieur de l'enfant, avoir été présent dans la " "nouvelle" jungle " avant le 24 octobre 2016 au plus tard, être entré sur le territoire européen avant le 20 mars 2016 ${ }^{28}$. Ces conditions restrictives avaient considérablement réduit la portée du dispositif.

Ensuite, le Royaume-Uni s'est engagé à accueillir des mineurs qui n'ont pas d'attache au Royaume-Uni : ce sont les mineurs isolés étrangers, qui seraient accueillis au titre de l' «amendement Dubs» précité. C'est ce que prévoit l'article 3 du Traité relatif au «programme national de réadmission ». En 2016, l'application l' "amendement Dubs » avait connu un semi-échec puisque la mention « 3000 enfants réfugiés » accueillis qu'il contenait fut remplacée par « un certain nombre d'enfants réfugiés non accompagnés ${ }^{29}$ 
accueillis. Le Royaume-Uni s'est engagé, à l'occasion de la signature du Traité, d'accueillir 480 mineurs, à condition d'y soustraire les 220 mineurs qui auraient déjà été accueillis ${ }^{30}$. $\mathrm{Au}$ final, il ne resterait que 260 places disponibles pour bénéficier de cet amendement. Alors que le guide d'application qui contenait les critères d'éligibilité à l'amendement fixait une date limite d'arrivée sur le territoire européen au 20 mars 2016, cette date a été repoussée au 18 janvier 2018. Le 20 janvier, alors que les modalités d'identification de ces mineurs n'étaient pas encore connues (elles ne le sont toujours pas), Gérard Collomb a réagi aux « arrivées de mineurs non accompagnés à Calais constatées depuis la signature du Traité », en affirmant qu'aucun mineur arrivé après le 18 janvier ne serait éligible au transfert et que les identifications ne se dérouleraient de toute manière pas à Calais. Gérard Collomb a justifié ces premières limitations par « la volonté ferme d'éviter toute reconstitution de campements à Calais et de maintenir le plus haut niveau possible de sécurisation de la frontière " ${ }^{31}$. C'est l'éternelle peur de l' " appel d'air ", formule qu'avait utilisée Nicolas Sarkozy pour justifier la fermeture du centre de Sangatte lorsqu'il était ministre de l'Intérieur. Il y avait a priori une centaine de mineurs à Calais. Depuis la signature du Traité, c'est au minimum 150 mineurs supplémentaires qui y seraient arrivés 32 .

Pour synthétiser, les seuls engagements formels et concrets du Royaume-Uni dans le cadre du Traité consistent à réduire ses délais relatifs à la procédure du regroupement familial des mineurs étrangers dans le cadre du règlement Dublin III - on ignore d'ailleurs le nombre qui sera accueilli à ce titre - et à accueillir un nombre de 260 mineurs isolés étrangers dans le cadre de l' "amendement Dubs". On est en droit, à l'aune de ces engagements peu ambitieux, de parler d'épiphénomène. La Haute Cour de justice britannique elle-même avait reconnu, par un arrêt du 29 janvier 2016, que l'application stricte des dispositions du règlement Dublin, promue par le secrétaire d'État à l'Intérieur pour refuser l'entrée au Royaume-Uni à quatre jeunes mineurs syriens isolés, était incompatible avec la Convention européenne des droits de l'homme, épinglant par la même occasion la France pour sa carence dans la mise en œuvre d'une telle procédure (pour toute l'année 2015, selon les juges, la France n'avait transféré que quatre demandes d'asile au Royaume-Uni) ${ }^{33}$.

L'insistance du Traité à propos des engagements britanniques vis-à-vis du règlement Dublin n'est pas fortuite : elle intervient en effet dans le contexte du Brexit qui devrait être effectif au 29 mars 2019. Ce texte, d'apparence seulement bilatérale, dévoile pourtant nombre d'indices, certes ambigus, sur la place future du Royaume-Uni au sein de l'Union européenne, et sur le point de vue de cette dernière concernant la coopération francobritannique.

\section{$2 \%$ - Des ambiguiités aux consonances européennes}

Si le traité de Sandhurst ne règlera certainement pas la question des nombreux exilés qui souhaitent rejoindre le Royaume-Uni, il présente un intérêt d'un tout autre ordre : au regard de la situation dans laquelle le Royaume-Uni est actuellement vis-à-vis de l'Union européenne, c'est-à-dire une procédure de retrait qui devrait être effectif lors du premier trimestre de l'année 2019, ce texte souffre d'un certain nombre d'incohérences et de contradictions qui mettent en lumière la stratégie britannique en matière migratoire à l'aune de cette même procédure. Nul ne sait encore exactement quelle sera la nature de la relation qu'entretiendra le Royaume-Uni avec l'Union et dans quels domaines le premier 
acceptera de continuer à mettre ses compétences en commun avec la seconde. Si les discussions ont commencé entre chefs d'État et de gouvernement à propos de la libre circulation ou du marché intérieur, rien n'a encore été négocié, semble-t-il, concernant la politique d'asile et d'immigration de l'Union. Par son rédigé et sa formulation, le traité de Sandhurst est une ébauche de ce qui pourrait être les ambitions britanniques concernant certaines prérogatives dont il bénéficie grâce à cette politique, à laquelle il n'est que partiellement lié (A). De plus, ce traité lève le voile sur une autre ambiguïté et prouve qu'il répond parfaitement à la stratégie de l'Union européenne en matière migratoire (B).

\section{A./ - Un plan B en cas de Brexit dur}

30 Lors de la campagne électorale pour le référendum du 23 juin 2016 sur l'appartenance du Royaume-Uni à l'Union européenne, les ténors du Parti pour l'indépendance du RoyaumeUni (UKIP) ont martelé que le vote en faveur du « Leave » permettrait au Royaume-Uni de reprendre la main sur sa politique migratoire ${ }^{34}$. Dans le camp adverse, chez les partisans $\mathrm{du}$ " Remain », le règlement Dublin III était l'argument vedette pour démontrer l'intérêt britannique de se maintenir au sein de l'Union européenne ${ }^{35}$. En effet, le règlement Dublin III, qui est un des rares textes européens à avoir obtenu le déclenchement de la clause d'opt-in du Royaume-Uni, est un outil indispensable à la politique britannique d'immigration. Lui permettant de renvoyer les demandeurs d'asile vers leur premier pays de transit sans avoir à en accueillir autant à ce titre, il permet au Royaume-Uni d'être le pays qui profite le plus des politiques européennes d'immigration tout en y participant à moindre frais. Néanmoins, la victoire du «Leave » lors du référendum change la donne. Théoriquement, lorsque le Brexit sera effectif, le Royaume-Uni ne pourra plus bénéficier des avantages du règlement Dublin auquel il tenait tant. Le traité de Sandhurst constitue un palliatif à cette perte d'avantages.

31 Premièrement, le traité de Sandhurst se révèle être une profession de foi britannique en faveur du règlement Dublin, alors que les accords franco-britanniques précédents ont consisté à le stériliser. Dès le préambule, le Traité affirme sa parfaite intelligence avec la législation européenne, en garantissant « que rien dans le présent traité ne devrait porter atteinte a) au maintien de l'application des obligations existantes des Parties en vertu du régime d'asile européen commun (RAEC), en particulier le règlement $n^{\circ}$ 604/2013 du Parlement européen et du Conseil du 26 juin 2013 établissant les critères et mécanismes de détermination de l'État membre responsable de l'examen d'une demande de protection internationale présentée dans l'un des États membres par un ressortissant d' un pays tiers ou un apatride (refonte)». Alors que le Traité ne respecte pourtant pas la législation européenne, une telle mention étonne d'autant plus qu'elle est inédite. Il est fait mention du Brexit, puisqu'il est précisé que le Traité ne portera pas atteinte «aux relations futures du Royaume-Uni avec l'Union européenne ».

Le paragraphe suivant prend une précaution supplémentaire, en ajoutant «que, dans la mesure où les dispositions du présent traité se rapportent au règlement 604/2013, les Parties confirment que : a) rien dans le présent traité n'est destiné à affecter la mise en œuvre légale du règlement 604/2013 par l'une des Parties ; et b) ces dispositions ne s'appliquent que lorsque les deux Parties participent au règlement 604/2013 ». A fortiori, l'article 2 est entièrement consacré au respect des dispositions du règlement Dublin III par le Royaume-Uni et la France : ces derniers « réitèrent leur obligation d'assurer le fonctionnement efficace du règlement 604/2013 tant que les deux Parties participent au 
règlement 604/2013 ». L'article 2 perfectionne même le règlement Dublin en écourtant ses délais, notamment ceux relatifs à la demande de prise en charge et à l'organisation du transfert des mineurs isolés étrangers, qui passent respectivement de trois mois à dix jours et de six mois à quinze jours.

Sachant que la sortie du Royaume-Uni de l'Union européenne sera effective à partir du 29 mars 2019, il est permis de se demander quel était l'intérêt pour les Britanniques de signer un traité qui prend tant de soin à souligner et à garantir le respect du règlement Dublin III dans le cadre de la coopération bilatérale. En effet, si ce retrait de l'Union comprend également l'arrêt de la participation britannique à l'application du règlement Dublin III, la signature du Traité perd tout son sens : soit il ne s'appliquerait que pour une durée d'un peu plus d'un an, soit la moitié du Traité deviendrait ineffective. Un traité n'étant pas signé pour une si courte durée ou pour être amputé de la moitié de ses dispositions un an plus tard, ce scrupule à garantir l'application du droit de l'Union européenne pourrait être révélateur d'une stratégie de la part du Royaume-Uni qui, très attaché au règlement Dublin, souhaiterait démontrer son envie de poursuivre son application. Une preuve supplémentaire allant dans le sens de cette hypothèse se trouve dans le préambule du Traité : fait quasiment révolutionnaire dans l'histoire de la coopération franco-britannique, ce traité a fait l'objet d'une validation auprès de la Commission européenne. En effet, comme le prévoit l'article 36 du règlement Dublin III :

«1. Les États membres peuvent établir entre eux sur une base bilatérale des arrangements administratifs relatifs aux modalités pratiques de mise en œuvre du présent règlement afin d'en faciliter l'application et d'en accroître l'efficacité. Ces arrangements peuvent porter sur :

des échanges d'officiers de liaison;

une simplification des procédures et un raccourcissement des délais applicables à la transmission et à l'examen des requêtes aux fins de prise en charge ou de reprise en charge de demandeurs d'asile. »

Le paragraphe 2 de cet article confère à la Commission européenne un pouvoir de surveillance vis-à-vis de ces arrangements administratifs pour vérifier leur compatibilité au regard du droit de l'Union européenne. Le Traité comprend les dispositions inclues dans les sous-paragraphes a) et b), mais les accords précédents conclus entre le RoyaumeUni et la France, qui s'inscrivaient pourtant bien « dans le cadre du règlement Dublin III » ${ }^{36}$, comme le précise notamment l'accord du 20 août 2015, n'ont jamais fait l'objet d'une quelconque validation par la Commission européenne ${ }^{37}$. Il étonnant que, pour le traité de Sandhurst, les démarches prévues par l'article 36 aient été effectuées auprès de la Commission européenne, alors que cette dernière a elle-même suggéré de supprimer ce pouvoir de contrôle et de validation dans sa proposition de refonte du règlement Dublin III le 4 mai $2016^{38}$. Cette inclinaison du Royaume-Uni à vouloir démontrer sa bonne volonté auprès des institutions européennes témoigne une fois de plus de sa volonté de poursuivre l'application du règlement Dublin III, même après le Brexit.

Si les États membres et les institutions européennes refusent cette participation au-delà, le traité de Sandhurst semble avoir prévu une solution de repli : les articles 4 et 7 susdits du Traité prévoient qu'en cas de « pressions créées par les ressortissants de pays tiers qui n'ont pas de base légale de séjour sur le territoire français ", c'est-à-dire ceux qui n'ont pas déposé de demande d'asile - pour toutes les raisons que l'on connaitt (volonté de gagner le Royaume-Uni, peur d'être " dublinés », etc.) -, le Royaume-Uni apportera son aide à la France par diverses mesures de retour "vers les pays d'origine et de transit " prévues par l'article 7. Ironiquement, le traité de Sandhurst pourrait s'avérer être un 
texte encore plus avantageux pour le Royaume-Uni que le règlement Dublin III lui-même : alors que ce dernier permet au Royaume-Uni de renvoyer les exilés présents sur le territoire britannique vers un pays par lequel ils ont transité, le Traité lui permet de renvoyer les exilés présents non pas sur le territoire britannique, mais sur le territoire français, non seulement dans un pays par lequel ils ont transité - à l'instar du règlement Dublin -, mais également dans leur pays d'origine!

Le Royaume-Uni préparerait donc une sortie «en douceur » de l'Union européenne, du moins concernant sa politique d'asile et d'immigration. Toutefois, le Traité révèle davantage que cela : à sa lecture, il indique que le Royaume-Uni se prépare aussi ce que les autres États membres ainsi que les institutions lui refusent sa participation à la politique d'asile et d'immigration européenne après le Brexit. C'est ce que révèle l'article 6 du Traité, qui concerne la création du Joint Information and Coordination Centre, soit en français le Centre commun d'information et de coordination (C.C.I.C.), dans un cadre purement bilatéral, indépendamment de toute appartenance à l'Union européenne de l'un des deux États. Trois objectifs sont donnés à ce nouveau centre. Le premier consiste en la gestion et la prévention des menaces à l'ordre public sur les infrastructures de transport transfrontalières dans le Nord et le Pas-de-Calais. Le deuxième concerne la gestion de crises "en cas de pressions migratoires aiguës" : le C.C.I.C. servirait de «plateforme d'aide à la prise de décisions pour les préfets départementaux compétents ». Le dernier objectif vise le renforcement de la coopération entre les services des deux pays concernant « le soutien à l'action de lutte contre les opérations des filières de contrebande, des trafiquants d'êtres humains et des réseaux criminels dans le Nord et le Pas-de-Calais ». Là encore, la mise en place de ce C.C.I.C. semble être une véritable « issue de secours » pour les Britanniques en cas de Brexit « dur ».

Si le retrait de l'Union se déroule de cette manière, les États membres et les institutions européennes pourraient refuser que le Royaume-Uni continue à participer au règlement no 603/2013 du Parlement européen et du Conseil de l'Union européenne du 26 juin 2013 relatif à la création d'Eurodac. Grâce à la prise d'empreintes digitales et à la collecte de données relatives au profil du demandeur d'asile, les pays de l'Union peuvent vérifier si un demandeur d'asile ou un ressortissant étranger sur son territoire a déjà formulé une demande dans un autre pays de l'Union. Ainsi, un État membre est responsable d'une demande d'asile dans deux cas : si un exilé a été contrôlé avec prise d'empreintes digitales au moment du franchissement de sa frontière ou s'il y a séjourné " pendant une période continue d'au moins cinq mois avant d'introduire sa demande de protection internationale $\aleph^{39}$. Le Royaume-Uni est également attaché à la gigantesque base de données que constitue le système Eurodac, d'autant plus que le Conseil de l'Union a approuvé un mandat en vue de négociations avec le Parlement européen sur sa refonte. Comme le précise un communiqué du 9 décembre 2016 du Conseil, cette réforme prévoit « d'adapter et de renforcer le système Eurodac et d'en élargir le champ d'application, en vue de faciliter les retours et de contribuer à la lutte contre les migrations irrégulières ». Plus précisément, les États membres auront la possibilité « de stocker et de rechercher des données appartenant à des ressortissants de pays tiers ou à des apatrides qui ne sont pas demandeurs d'une protection internationale et qui se trouvent en séjour irrégulier sur le territoire de l'Union européenne, de manière à ce qu'ils puissent être identifiés à des fins de retour et de réadmission $»^{40}$. Le Royaume-Uni n'est évidemment pas insensible à un tel instrument. La création du C.C.I.C., dont l'un des principaux objectifs consiste à la coopération des services en matière d' "action de lutte contre les opérations des filières 
de contrebande, des trafiquants d'êtres humains et des réseaux criminels " serait donc un bon moyen pour le Royaume-Uni, en cas de Brexit "dur ", de pérenniser son accès à Eurodac via sa coopération bilatérale avec la France, qui, elle, participera toujours à l'application de ce règlement après le 29 mars 2019.

Le Royaume-Uni a donc pris nombre de précautions en prévoyance du Brexit ; pourtant, il n'a toujours pas communiqué sur sa future participation éventuelle à la politique européenne en matière d'asile et d'immigration. Car il reste une inconnue : la position des États membres et des institutions de l'Union concernant cette hypothétique participation après le Brexit. La France ne semble pas y être opposée, la signature de ce traité la contraint de toutes les façons à assumer une politique d'asile et d'immigration étroitement liée à celle du Royaume-Uni. Concernant les instances de l'Union, le Traité précise que son texte a été soumis au contrôle de la Commission européenne. Alors que l'Union européenne s'est enfermée dans un mutisme total concernant la coopération franco-britannique pendant des dizaines d'années, alors même qu'elle a élaboré une politique d'asile et d'immigration censée primer sur celle de ses États membres, elle semble aujourd'hui prendre explicitement mais discrètement position en faveur de la poursuite de cette coopération. Et pour cause, le Royaume-Uni et la France ne font rien de plus en matière migratoire que ce que fait l'Union européenne depuis des années.

\section{B./ - Un traité « eurocompatible »}

39 Le dernier article de fond du Traité, l'article 8, intitulé « Action conjointe dans les pays d'origine de l'immigration clandestine », aurait pu tout aussi bien s'appeler « Last but not least ». Il est une partie incontournable du texte, révélatrice de sa quintessence. Son titre provoque d'abord une certaine surprise : alors que tout le Traité expose des mesures concernant les exilés, censées améliorer le traitement de leur demande d'asile, l'article 8 parle d' " action conjointe dans les pays d'origine de l'immigration clandestine ». Il n'est fait nulle autre part mention, dans le Traité, d' « immigration clandestine ». Il ne donne pas non plus de définition à ce qu'il considère comme l' "immigration clandestine », ce qui laisse à penser que les exilés qui sont au cœur des sept articles précédents sont considérés comme étant des protagonistes de l' "immigration clandestine", soit des « immigrés clandestins ». Le choix de ces mots n'est pas innocent, et le message envoyé par cet article se veut clair : les exilés présents à la frontière franco-britannique souhaitant gagner le Royaume-Uni sont des «illégaux », soit des individus étant entrés sur le territoire français sans avoir en leur possession des documents délivrés par l'administration française, c'est-à-dire un visa ou un titre de séjour. Cette désignation de l' «immigration clandestine», dont le poids politique ne peut être ignoré par les signataires du Traité, témoigne de la volonté de suspicion et de criminalisation des exilés bloqués à la frontière entre les deux pays. Le contenu de l'article confirme cette inclinaison en disposant que l'accord trouvé entre les deux États vise « à affecter les flux migratoires aux stades antérieurs, dans les pays d'origine et de transit ». La rédaction de la formule "affecter les flux migratoires aux stades antérieurs", correspond tout simplement, en termes plus triviaux, à « couper le mal à la racine ».

Pour ce faire, la première mesure mise en œuvre consistera à réaliser des « interventions destinées à lutter contre la traite et l'immigration clandestine, y compris des programmes d'information dissuasifs ciblant les populations susceptibles d'envisager des mouvements illégaux ", soit la mise en place de véritables campagnes d'information dissuasives à 
l'égard des exilés dans leur pays d'origine. Le deuxième alinéa prévoit quant à lui « des mesures, y compris des programmes visant à renforcer la réintégration des migrants qui ont été renvoyés dans leur pays d'origine ou dans un pays où ils sont légalement admissibles ». Les deux premiers alinéas de l'article 8 touchent donc à deux aspects : un aspect préventif, la communication pour dissuader l' « immigration irrégulière », puis un aspect palliatif, la réadmission des exilés dans leur pays d'origine.

La mise en place de campagnes d'information dissuasives, si elles sont assez nouvelles dans le cadre de la coopération franco-britannique, n'ont rien d' «innovant» sur le territoire européen. En effet, plusieurs États d'Europe ont déjà songé voire mis en application des campagnes d'information de ce genre. Parmi les premiers d'entre eux, on compte le Royaume-Uni lui-même qui, en 2013, avait songé à lancer une grande campagne d'information en Roumanie et en Bulgarie, pour persuader les potentiels immigrants d'éviter d'aller au Royaume-Uni ${ }^{41}$. Autre pays européen à considérer ce genre de campagne, le Danemark a publié en 2015, dans les journaux étrangers, notamment libanais, une campagne d'information à destination des demandeurs d'asile et plus généralement des immigrés pour les dissuader de gagner le territoire danois ${ }^{42}$. En collaboration avec l'Organisation internationale pour les migrations (O.I.M.), l'Italie a également lancé, en 2016, une grande campagne d'information sur Internet, intitulée «Aware Migrants» (« Avertir les migrants »), pour alerter les candidats au départ sur les «dangers du voyage " jusqu'aux côtes italiennes ${ }^{43}$. La Suède a soutenu, en collaboration avec le Fonds des Nations unies pour l'enfance (UNICEF), une initiative de l'O.I.M. consistant à organiser des "activités de sensibilisation sur les risques associés à la migration des enfants» dans plusieurs États d'Afrique de l'Ouest, notamment en déployant des banderoles avec le message «Ensemble pour la protection des enfants contre l'immigration irrégulière" lors de matchs de football ${ }^{44}$. Les campagnes d'information prévues dans l'article 8 du traité de Sandhurst ne provoqueront donc pas une bronca de la part des États européens, et encore moins de l'Union européenne.

C'est ce qu'a expliqué Cecilia Malmström, alors commissaire européen aux Affaires intérieures, en 2010, lors d'une séance de réponses aux questions parlementaires : « La prévention de l'immigration clandestine est un des piliers de l'Approche globale sur les migrations, politique extérieure de l'Union européenne en matière d'immigration. »Cette politique, explique le Commissaire, se matérialise par «le financement de campagnes d'information et de prévention», considéré comme "une composante essentielle de la mise en œuvre de cette priorité $»^{45}$. La Commission européenne avait d'ailleurs lancé un programme thématique Migration et Asile dans le cadre de son Instrument de financement de la coopération au développement, dont les mesures figurent dans le règlement $\mathrm{n}^{\circ}$ 1906/2005 portant établissement d'un instrument de financement de la coopération au développement, qui ne s'applique plus depuis le 31 décembre 2013. Le programme couvrait des domaines d'activité comme «la lutte contre l'immigration clandestine et l'aide à la réadmission des immigrants clandestins, y compris entre pays tiers, et en particulier la lutte contre les filières clandestines d'immigration et la traite d'êtres humains, les mesures visant à décourager l'immigration clandestine et à sensibiliser les personnes aux risques qu'elle comporte $[. ..] »^{46}$. Concernant le volet palliatif, c'est-à-dire la réadmission, l'Union européenne, parfois en collaboration avec l'O.I.M., a déjà mis en place l'expérience pour des ressortissants burkinabés, camerounais, tchadiens, gambiens, ghanéens, bissaoguinéens, guinéens, maliens, mauritaniens, nigériens, nigérians, sénégalais, ivoiriens et libyens ${ }^{47}$, mais aussi marocains et tunisiens ${ }^{48}$. 

commissaire Malmström conclut sa réponse ainsi : «La prévention et la réduction de l'immigration clandestine demeurant une priorité pour l'Union européenne, la Commission continuera à développer à l'avenir ce genre d'initiatives. $»^{49}$ Concernant les campagnes d'information visant à dissuader l'immigration et la réadmission des exilés, Royaume-Uni, France, États européens et Union européenne sont donc sur la même longueur d'ondes. Sur ce point, il est alors évident que le traité de Sandhurst a bénéficié de bienveillance certaine de la part de la Commission européenne.

Marion Tissier-Raffin identifie, parmi les politiques d'asile et d'immigration mises en œuvre récemment, « des caractéristiques identiques et novatrices par rapport à celles des années 90 ", qui reposent "sur la mise en place d'un arsenal complexe et massif pour bloquer les demandeurs d'asile aux frontières extérieures de l'Union européenne ». D'après elle, la première caractéristique commune concerne le renforcement de «la coordination des polices nationales de contrôle des frontières extérieures au niveau communautaire $\aleph^{50}$. Cette disposition se matérialise par l'adoption, en 2004, du règlement $\mathrm{n}^{\mathrm{o}}$ 2007/2004 du Conseil de l'Union européenne portant création d'une Agence européenne pour la gestion de la coopération opérationnelle aux frontières extérieures des États membres de l'Union européenne, abrogé en 2016 par le règlement n² 2016/1624 du Parlement européen et du Conseil de l'Union européenne relatif au corps européen de garde-frontières et de garde-côtes. Les accords successifs conclus entre le Royaume-Uni et la France ont également prévu des mesures de ce genre : l'accord administratif d'Évian de 2009 prévoyait « la mobilité d'agents de l'Agence des frontières du Royaume-Uni (UK Border Agency) afin qu'ils s'installent dans le nord de la France et ainsi améliorer l'efficacité de la gestion du personnel de contrôle à la frontière commune $»^{51}$. Le traité de Sandhurst, quant à lui, coordonne les polices britannique et française de contrôle des frontières au niveau bilatéral, en entérinant la création du C.C.I.C., déjà prévue par la déclaration du 20 août 2015, qui réunira «en son sein les différents services de sécurité français, les personnels chargés du contrôle aux frontières du Royaume-Uni, l'Agence nationale de lutte contre la criminalité, ainsi que les autres agences britanniques $»^{52}$.

Pour Marion Tissier-Raffin, la deuxième caractéristique consiste en « des partenariats [...] conclus avec les pays de transit pour que leurs polices soient formées au contrôle de leurs frontières $~^{53}$, via l'adoption, en 2004, du règlement no 491/2004 du Parlement européen et du Conseil de l'Union européenne établissant un programme d'assistance technique et financière en faveur de pays tiers dans le domaine des migrations et de l'asile (AENEAS), abrogé par le règlement $n^{\circ}$ 1905/2006 susdit. Ce critère se retrouve dans le dernier alinéa de l'article 8 du traité de Sandhurst, qui prévoit un renforcement de «la coopération opérationnelle et [des] capacités régionales de gestion des frontières dans les pays d'origine et de transit pour lutter contre la traite des êtres humains, le trafic de migrants clandestins, la criminalité organisée en matière d'immigration et les questions liées à l'esclavage moderne, et assurer une protection appropriée des victimes ». Pour endiguer l' « immigration clandestine », le Royaume-Uni et la France s'engagent à apporter un réel soutien aux pays d'origine des exilés pour les empêcher de quitter leur propre pays, ce qu'interdisent formellement l'article 2 du paragraphe 2 du protocole $n^{\circ} 4$ annexé à la Convention européenne des droits de l'homme ainsi que l'article 13 de la Déclaration universelle des droits de l'homme, qui garantissent à tout individu le droit de quitter un pays, y compris le sien. Cette mesure est d'un genre nouveau. Elle n'est en soi ni une réadmission, ni un refoulement : elle consiste à bloquer des individus à leurs propres

La Revue des droits de l'homme , Actualités Droits-Libertés 
frontières nationales pour les empêcher de quitter leur propre pays. En août dernier, Emmanuel Macron recevait à Paris plusieurs dirigeants africains pour les convaincre d'installer des centres d'enregistrement des demandeurs d'asile en Afrique, notamment au Tchad, pour éviter aux exilés «de prendre des risques inconsidérés $»^{54}$, une proposition qui va dans le même sens que l'engagement franco-britannique contenu dans l'alinéa 3 de l'article 8 du Traité. Cette proposition n'a rien de neuf : lors du Conseil européen de Séville de 2002, le Premier ministre britannique Tony Blair avait proposé « la création de "zones de protection régionale" permettant d'assurer la sécurité des candidats à l'asile dans leur région d'origine ainsi que la mise sur pied de "centres de traitement de transit" autorisant un examen à distance des demandes d'asile " ${ }^{55}$. Écartées cette année-là, ces propositions furent validées quelques années plus tard, lors du sommet européen de La Haye de 2004 ${ }^{56}$. La suggestion d'Emmanuel Macron sera certainement compliquée à réaliser, les États africains n'y étant guère favorables. Par exemple, le ministre des Affaires étrangères tchadien, Hissein Brahim Taha, avait rejeté l'idée par crainte de voir se former dans son pays... un « appel d'air " ${ }^{57}$.

La troisième caractéristique relevée par Marion Tissier-Raffin concerne « des accords de réadmission [...] conclus avec ces mêmes pays [d'origine] pour qu'ils reprennent les étrangers refoulés ${ }^{58}$, via des accords communautaires négociés avec les pays d'origine des ressortissants. Dans le cas du traité de Sandhurst, cette politique de retours organisés bénéficie d'un article entier, l'article 7, ainsi que de plusieurs mentions tout au long du texte. Pour Marion Tissier-Raffin, «l'objectif de ces politiques est donc très clair : il ne s'agit plus seulement de dissuader les migrants économiques de pénétrer sur le sol européen mais de bloquer l'arrivée de tout étranger, quel que soit le motif de son départ, aux frontières extérieures de l'Union européenne, voire en amont de ces frontières quand le blocage s'opère aux frontières des pays de transit $"^{59}$. À l'inverse, dans le cadre de la coopération franco-britannique, il ne s'agit plus seulement de bloquer les exilés à la frontière séparant le Royaume-Uni de la France, mais de dissuader les exilés, jusque dans leur pays d'origine, d'atteindre cette frontière.

*

Traité entre le gouvernement du Royaume-Uni de Grande-Bretagne et d'Irlande du Nord et le gouvernement de la République française relatif au renforcement de la coopération pour la gestion coordonnée de leur frontière commune, entré en vigueur le 1er février 2018

\section{NOTES}

1. Nathalie RAULIN, « Macron ni de droite ni de gauche... ni hors système », Libération, 3 fév. 2017 [http://www.liberation.fr/politiques/2017/02/03/macron-ni-de-droite-ni-degauche-ni-hors-systeme_1546275].

2. Clémence BAUDUIN, « Emmanuel Macron menace David Cameron d'autoriser le passage des migrants au Royaume-Uni en cas de Brexit », Radio Télé Luxembourg, 3 mars 2016 
[http://www.rtl.fr/actu/international/ emmanuel-macron-menace-david-cameron-dautoriser-le-passage-des-migrants-au-royaume-uni-en-cas-de- brexit-7782166924]. 3. Tristan QUINAULT-MAUPOIL, « Migrants : Cazeneuve recadre Macron et l'accuse de vouloir faire "le buzz" », Le Figaro, 4 mars 2016 [http://www.lefigaro.fr/politique/le-scan/ couacs/2016/03/04/25005-20160304ARTFIG00130-migrants-cazeneuve-recadre-macronet-l-accuse-de-vouloir-faire-le-buzz.php].

4. Emmanuel JARRY, Sophie LOUET, Estelle SHIRBON, « "Une sortie est une sortie”, dit Macron à Theresa May », Reuters, 21 fév. 2017 [http://fr.reuters.com/article/topNews/ idFRKBN1601K9].

5. Ashley COWBURN, «Theresa May admits Calais border controls could be up for discussion after Macron's election victory », The Independent, 8 mai 2017 [http://www.independent.co.uk/ news/uk/politics/calais-border-control-talks-immigration-theresa-may-emmanuelmacron-victory-french-president-a7724181.html].

6. Présidence de la République française, «Conférence de presse avec Theresa May à Sandhurst pour le $35^{\mathrm{e}}$ sommet franco-britannique », 20 janv. 2018 [http://www.elysee.fr/ conferences-de-presse/article/conference-de-presse-avec-theresa-may-a-sandhurstpour-le-35-sommet-franco-britannique/].

7. Ministre de l'Intérieur : Éric Besson.

8. Ministre de l'Intérieur : Bernard Cazeneuve.

9. Ministre de l'Intérieur : Gérard Collomb.

10. Antoine GUÉRIN, Aspects juridiques européens de la situation migratoire à Calais, collection « Les mémoires de l'Équipe de droit international, européen et comparé », Université Jean Moulin Lyon 3, 2017, pp. 58-75 [http://ediec.univ-lyon3.fr/fileadmin/ medias/Documents_EDIEC/Memoires_EDIEC/Mem_16_EDIEC_GUERIN_Antoine-2017.pdf]. 11. Olivier PECQUEUX, « À quoi serviront les 50 millions d'euros promis par les Britanniques? », La Voix du Nord, 31 janv. 2018 [http://www.lavoixdunord.fr/308794/ article/2018-01-31/quoi-serviront-les-50-millions-d-euros-promis-par-les-britanniques]. 12. L'assignation à résidence consiste à maintenir un étranger dans un endroit précis. 13. La rétention administrative consiste à maintenir un étranger qui fait l'objet d'une mesure d'éloignement du territoire français dans un lieu fermé et surveillé en attendant son renvoi forcé.

14. C.J.U.E., $2^{\mathrm{e}}$ ch., Policie ČR contre Salah Al Chodor, Ajlin Al Chodor et Ajvar Al Chodor, 15 mars 2017, C-528/15.

15. Marie-Laure BASILIEN-GAINCHE, « Suite française. Les implications de l'affaire Al Chodor concernant la rétention des demandeurs d'asile dublinés », European Database of Asylum Law, 27 nov. 2017 [http://www.asylumlawdatabase.eu/en/journal/suite-française-lesimplications-de-l'affaire-al-chodor-concernant-la-rétention-des].

16. C.E., $2^{\mathrm{e}}$ et $7^{\mathrm{e}}$ ch., avis, 19 juillet $2017, \mathrm{n}^{\mathrm{e}} 408919$.

17. Jean-Marc PASTOR, «Le Sénat modifie la proposition de loi relative au régime d'asile européen », Dalloz actualité, 30 janv. 2018 [https://www.dalloz-actualite.fr/flash/senatmodifie-proposition-de-loi-relative-au-regime-d-asile-europeen\#.WntfRWZeg1h].

18. Sénat, proposition de loi permettant une bonne application du régime d'asile europé en, $\mathrm{n}^{\mathrm{0}} 219,17$ janv. 2018, p. 5 [http://www.senat.fr/leg/ppl17-219.pdf].

19. Alors que le renvoi d'un «dubliné » dans le premier pays par lequel il rentré dans l'Union européenne doit être le dernier critère envisagé par les États membres, cela n'est pratiquement jamais le cas.

20. Déclaration franco-britannique sur l'immigration, 6 juil. 2009, p. 1. 
21. Déclaration commune des ministres de l'Intérieur français et britannique portant sur la coopération entre la France et le Royaume-Uni, « Faire face à la pression migratoire à Calais », 20 août 2015, § 16 (I).

22. C.E.D.H., G.C., Hirsi Jamaa et autres contre Italie, 23 fév. 2012, no 27765/09 et C.E.D.H., Sharifi et autres contre Italie et Grèce, 21 oct. 2014, no 16643/09.

23. C.E.D.H., communiqué du greffier, « La Cour européenne des droits de l'homme accorde la demande de mesures provisoires introduite par 11 afghans ", 18 nov. 2008. 24. Olivier PECQUEUX, op. cit.

25. Ministère de la Justice, circulaire interministérielle relative à la mobilisation des services de l'État auprès des conseils départementaux concernant les mineurs privés temporairement ou définitivement de la protection de leur famille et les personnes se présentant comme tels, 25 janv. 2016, annexe 12.

26. Code de l'entrée et du séjour des étrangers et du droit d'asile, art. L. 741-3.

27. C.E., 21 avr. 2017, nº 409806.

28. Gouvernement du Royaume-Uni, Bureau de l'Intérieur, « Guidance: Implementation of section 67 of the Immigration Act 2016 in France », 8 nov. 2016, p. 6.

29. Gouvernement du Royaume-Uni, Immigration Act 2016, chap. 19, part. 5, § 67, « Unaccompanied

refugee children: relocation and support ».

30. Gouvernement du Royaume-Uni, « Fact sheet on the UK's support for asylum-seeking and refugee children in Europe », 19 janv. 2018 [https://

homeofficemedia.blog.gov.uk/2018/01/19/fact-sheet-on-the-uks-support-for-asylumseeking-and-refugee-children-in-europe/].

31. Ministère de l'Intérieur, « Transfert de mineurs non accompagnés vers le RoyaumeUni », communiqué de presse, 20 janv. 2018.

32. L'Auberge des migrants, «Communiqué sur la situation des mineurs et les voies de passage (Sandhurst, Dubs) », communiqué de presse, 22 janv. 2018 [http://

www.laubergedesmigrants.fr/fr/communique-situation-mineurs-a-calais-traite-desandhurst-lamendement-dubs/].

33. Haute Cour de justice (Royaume-Uni), chambre de l'Immigration et de l'Asile, The Queen on the application of ZAT, IAJ, KAM, AAM, MAT, MAJ and LAM v. Secretary of State for the Home Department, 29 janv. 2016, JR/15401/2015 et JR/15405/2015, §§ 23 et 58, traduit de l'anglais par Maud ANGLIVIEL, « Une juridiction britannique ordonne l'admission au Royaume-Uni de mineurs isolés vivant dans la "jungle" de Calais », La Revue des droits de l'homme, $1^{\mathrm{er}}$ mars 2016 [https://revdh.revues.org/1807? lang=en\#ftn13].

34. Rowena MASON, Heather STEWART, « Nigel Farage's anti-migrant poster reported to police », The Guardian, 16 juin 2016 [https://www.theguardian.com/politics/2016/jun/16/nigelfarage-defends-ukip-breaking-point-poster-queue-of-migrants].

35. Hugo DIXON, « Think leaving the EU would strengthen Britain's borders? Take a closer look », The Guardian, 22 sept. 2015 [https://www.theguardian.com/commentisfree/2015/sep/22/ eu-borders-eurosceptics- britain-refugee-crisis-europe]

36. Déclaration commune des ministres de l'Intérieur français et britannique portant sur la coopération entre la France et le Royaume-Uni, « Faire face à la pression migratoire à Calais », 20 août 2015, § 24.

37. Les accords précédents dépassaient largement le cadre institué par l'article 36. Ils adoptaient des mesures autres que les échanges d'officiers de liaison, la simplification des procédures et le raccourcissement des délais. Théoriquement, si l'on s'en tient exclusivement au règlement Dublin III, ils sont dépourvus de base légale. 
38. Commission européenne, proposition de règlement du Parlement européen et du Conseil établissant les critères et mécanismes de détermination de l'État membre responsable de l'examen d'une demande de protection internationale introduite dans l'un des États membres par un ressortissant de pays tiers ou un apatride (refonte), $\operatorname{COM}(2016)$ 270 final, 4 mai 2016, pp. 79-80 [http://eur-lex.europa.eu/legal-content/FR/ TXT/PDF/? uri=CELEX:52016PC0270\&from=EN]

39. Parlement européen, Conseil de l'Union européenne, règlement (UE) nº 604/2013 établissant les critères et mécanismes de détermination de l'État membre responsable de l'examen d'une demande de protection internationale introduite dans l'un des États membres par un ressortissant de pays tiers ou un apatride (refonte), 26 juin 2013, art. 13-2, J.O.U.E., L 180, 26 juin 2013, p. 40.

40. Conseil de l'Union européenne, « Réforme du régime d'asile européen commun : le Conseil est prêt à entamer des négociations sur Eurodac ", communiqué de presse, 9 déc. 2016 [http://www.consilium.europa.eu/fr/press/press-releases/2016/12/09/asylumsystem/].

41. Rajeev SYAL, «Immigration: Romanian or Bulgarian? You won't like it here », The Guardian, 27 janv. 2013 [https://www.theguardian.com/uk/2013/jan/27/uk-immigration-romaniabulgaria-ministers].

42. Adam TAYLOR, « Refugee crisis: Denmark discourages asylum seekers with newspaper adverts in Lebanon ", The Independent, 8 sept. 2015 [http://www.independent.co.uk/news/world/ europe/refugee-crisis-denmark-discourages-asylum-seekers-with-newspaper-adverts-inlebanon-10490666.html].

43. Anne LE NIR, « En Italie, la campagne “Aware Migrants" veut dissuader les émigrés », Radio France internationale, 29 juil. 2016 [http://www.rfi.fr/emission/20160729-italiecampagne-aware-migrants-dissuasion-refugies-afrique-erythree-ethiopie].

44. O.I.M., « Les pays d'Afrique de l'Ouest sensibilisent la population aux risques de migration irrégulière des enfants et des jeunes », 17 nov. 2017, [https://www.iom.int/fr/ news/les-pays-dafrique-de-louest-sensibilisent-la-population-aux-risques-de-migrationirreguliere].

45. Parlement européen, questions parlementaires, 19 mars 2010, « Réponse donnée par $\mathrm{M}^{\text {me }}$ MALMSTRÖM au nom de la Commission » [http://www.europarl.europa.eu/sides/ getAllAnswers.do?reference=E-2010-0129\&language=ES\#def1].

46. Parlement européen, Conseil de l'Union européenne, règlement (CE) no 1905/2006 portant établissement d'un instrument de financement de la coopération au développement, 18 déc. 2006, art. 16, J.O.U.E., 27 déc. 2006, L 378, p. 55.

47. Service européen pour l'action extérieure, « Un an de partenariat U.E.-O.I.M. pour la protection et la réintégration des migrants en Afrique », 14 déc. 2017 [https:// eeas.europa.eu/headquarters/headQuarters-homepage/37767/un-de-partenariat-ue-oimpour-la-protection-et-la-réintégration-des-migrants-en-afrique_fr].

48. O.I.M. Maroc, « Organisation d'une table ronde dans le cadre du projet Mo-Tu-Se sous le thème : renforcer les solutions de réintégration au Maroc, Tunisie et Sénégal - mardi 20 décembre 2016 - Rabat », 22 déc. 2016 [https://morocco.iom.int/news/organisationd'une-table-ronde-dans-le-cadre-du-projet-mo-tu-se-sous-le-thème-renforcer-les]. 49. Parlement européen, questions parlementaires, 19 mars 2010, « Réponse donnée par $\mathrm{M}^{\mathrm{me}}$ MALMSTRÖM au nom de la Commission » [http://www.europarl.europa.eu/sides/ getAllAnswers.do?reference=E-2010-0129\&language=ES\#def1].

50. Marion TISSIER-RAFFIN, «Évaluer le droit par la science économique : essai d'application aux politiques européennes d'asile », in Véronique CHAMPEIL-DESPLATS (dir.) 
et Danièle Lоснак (dir.), À la recherche de l'effectivité des droits de l'homme, Presses universitaires de Paris-Nanterre, 2008, § 22 [http://books.openedition.org/pupo/1182]. 51. Déclaration franco-britannique sur l'immigration, 6 juil. 2009, p. 3.

52. Déclaration commune des ministres de l'Intérieur français et britannique portant sur la coopération entre la France et le Royaume-Uni, « Faire face à la pression migratoire à Calais », 20 août 2015, § 15 (IV).

53. Marion TISSIER-RAFFIN, op. cit.

54. Célian MACÉ, « Demandes d'asile : Macron défend ses “hot spots” au Niger et au Tchad », Libération, 28 août 2017 [http://www.liberation.fr/planete/2017/08/28/ demandes-d-asile-macron-defend-ses-hot-spots-au-niger-et-au-tchad_1592571]. 55. Denis DUEZ, L'Union européenne et l'immigration clandestine. De la sécurité intérieure à la construction de la communauté politique, Bruxelles, Éditions de l'université de Bruxelles, 2008, p. 114.

56. Idem, p. 118.

57. « À Paris, Idriss Déby obtient plus de 15 milliards d'euros de promesses d 'investissements », France 24, 9 sept. 2017 [http://www.france24.com/fr/20170909-parisidriss-deby-obtient-milliards-euros-promesses-investissements-crise-petrole].

58. Marion TISSIER-RAFFIN, op. cit.

59. Idem.

\section{RÉSUMÉS}

Le traité de Sandhurst s'ajoute à la longue liste des traités, protocoles et arrangements conclus entre le Royaume-Uni et la France depuis 1986. Au nombre de dix-neuf, tous poursuivent un but principal : restreindre, si ce n'est empêcher, l'accès des exilés au territoire britannique. Toujours plus rigoureux et coercitifs, indistinctement de l'étiquette politique du gouvernement qui les signe, ces textes aux contours légaux relativement flous sont à l'origine d'un régime juridique ad hoc qui concurrence voire neutralise les législations nationales, européennes et internationales en matière de droit des migrations et de droits de l'homme. Ce faisant, letraité valide et sacralise tous les codes et les fondements établis quinze ans plus tôt par le traité du Touquet. Nonobstant, il apporte un éclairage non négligeable, bien que très équivoque, quant à l'évolution de la coopération franco-britannique ainsi qu'aux relations entre le Royaume-Uni et l'Union européenne à la suite du Brexit .

\section{AUTEUR}

\section{ANTOINE GUÉRIN}

Doctorant en droit public à l'université Jean-Moulin Lyon III 